\title{
NÚCLEO DE ENFERMEIROS DE EDUCAÇÃO PERMANENTE DO PARANÁ: TRAJETÓRIA E CONTRIBUIÇÕES*
}

\author{
Priscila Meyenberg Cunha Sade ${ }^{1}$, Aida Maris Peres², João Vanderlei Machado Pedroso ${ }^{3}$, Liliana Müller \\ Larocca $^{4}$
}

\begin{abstract}
RESUMO: O objetivo deste estudo foi descrever a trajetória e contribuição na institucionalização da educação permanente nas organizações hospitalares desde sua fundação até os dias atuais. Pesquisa histórico-social, fundamentada na análise das fontes do acervo de um núcleo de enfermeiros do estado do Paraná, realizada entre o período de março de 2013 e janeiro de 2014. As transformações ocorridas nesse núcleo, ao longo de quase cinco anos, consolidaram-no como espaço efetivo para discussão e reflexão na construção de conhecimentos relacionados aos processos de educação nessas organizações hospitalares. Conclui-se que espaços como este possibilitam uma visão crítica dos movimentos históricos até os tempos atuais, que envolvem a Proposta da Educação Permanente em Saúde; auxiliam na compreensão do presente, de forma reflexiva; e, fornecem perspectivas futuras para a área de Enfermagem.

DESCRITORES: Enfermagem; História da enfermagem; Educação continuada em enfermagem; Pesquisa em administração de enfermagem.
\end{abstract}

\section{CENTER OF CONTINUING EDUCATION OF NURSES OF PARANÁ: HISTORY AND CONTRIBUTIONS}

ABSTRACT: The present study aimed to describe the history and contribution in the institutionalization of continuing education in hospital institutions from its beginnings up until the present day. Historical social research based on analysis of sources of the collection of a center of nurses in the state of Paraná, performed between March 2013 and January 2014. The transformations occurred in the referred center, over almost five years, turned it into an effective space for discussing and reflect on the construction of knowledge related to the education processes in hospital institutions. It is concluded that these spaces are suitable for the establishment of a critical view of historical movements until now related to the Proposal of Continuing Health Education; shed light on the current situation and provide a future outlook for the Nursing field.

DESCRIPTORS: Nursing, History of nursing; Continuing education in nursing; Research in nursing management.

\section{NÚCLEO DE ENFERMEROS DE EDUCACIÓN PERMANENTE DEL PARANÁ: TRAYECTORIA Y CONTRIBUCIONES}

RESUMEN: Estudio cuyo objetivo fue describir la trayectoria y la contribución para la institucionalización de la educación permanente en las organizaciones hospitalares de su fundación hasta los días actuales. La investigación histórico social fue fundamentada en el análisis de las fuentes del acervo de un núcleo de enfermeros del estado de Paraná, realizada entre el periodo de marzo de 2013 a enero de 2014. Los cambios ocurridos en ese núcleo, a lo largo de casi cinco años, lo consolidaron como espacio efectivo para discusión y reflexión en la construcción de conocimientos referentes a los procesos de educación en esas organizaciones hospitalares. Se concluye que espacios como este posibilitan una visión crítica de los movimientos históricos hasta los tiempos actuales, los cuales se relacionan a la Propuesta de la Educación Permanente en Salud; ayudan en la compresión del presente, de forma reflexiva; y, fornecen perspectivas futuras para el área de Enfermería.

DESCRIPTORES: Enfermería; Historia de la enfermería; Educación continuada en enfermería; Investigación en administración de enfermería.

\footnotetext{
*Artigo extraído da dissertação de mestrado intitulada: “Desenvolvimento de competências gerenciais dos enfermeiros pelos serviços de educação permanente". Universidade Federal do Paraná, 2013.

${ }^{1}$ Enfermeira. Mestre em Enfermagem. Docente de Enfermagem da Universidade Federal do Paraná. Curitiba, PR, Brasil. ${ }^{2}$ Enfermeira. Doutora em Enfermagem. Docente de Enfermagem da Universidade Federal do Paraná. Curitiba, PR, Brasil. ${ }^{3}$ Enfermeiro. Especialista em Educação Especial. Coordenador do Serviço de Educação Continuada do Hospital das Nações. Curitiba, PR, Brasil.

${ }^{4}$ Enfermeira. Doutora em Educação. Docente de Enfermagem da Universidade Federal do Paraná. Curitiba, PR, Brasil.
}

Autor Correspondente:

Priscila Meyenberg Cunha Sade

Universidade Federal do Paraná

R. Ângelo Cunico, 600 - 82220-047 - Curitiba, PR, Brasil

E-mail: priscila.sade@gmail.com
Recebido: $11 / 12 / 2015$

Finalizado: $29 / 04 / 2016$ 


\section{INTRODUÇÃO}

Para que as organizações hospitalares desenvolvam seus talentos, aumentem a competitividade e obtenham os melhores resultados nos negócios, faz-se necessário que implantem sistemas organizacionais que privilegiem não apenas conhecimentos técnicos instrumentais, mas também o desenvolvimento de competências ${ }^{(1)}$.

Neste contexto, a educação permanente nas organizações hospitalares começa a ser percebida como ferramenta auxiliar ao processo de qualificação, pois utiliza como princípios: competência profissional, motivação organizacional e avaliação contínua, que serão alcançados a partir de uma transformação do profissional de saúde para atuar de forma crítica e reflexiva no seu cotidiano ${ }^{(2)}$.

A Organização Pan-Americana de Saúde (OPAS) recomenda que haja um elemento que exerça a direção operacional do setor ou serviço de educação. Observa-se uma tendência da responsabilização dos enfermeiros pelo gerenciamento do serviço de educação, no qual possuem a incumbência de providenciar suporte organizacional e administrativo, bem como implantar uma cultura do trabalho de qualidade nas instituições hospitalares, a qual se inicia a partir de planejamento estratégico de gestão com a participação ativa e dialógica dos profissionais destas organizações ${ }^{(3-4)}$.

Para tanto, os profissionais enfermeiros envolvidos com educação precisam se manter integrados aos ambientes interno e externo e às políticas vigentes. A não observância desses aspectos gera conflitos e insatisfação entre enfermeiros de educação e os profissionais de enfermagem ou outras áreas, uma vez que acarreta tomada de decisões inadequadas e, por conseguinte, ineficiência e ineficácia dos programas de educação permanente ${ }^{(4-5)}$.

Nesta perspectiva, em consonância com as transformações ocorridas nos processos educativos nos últimos anos, que buscam fortalecer a reflexão na ação, o trabalho em equipes e a capacidade de gestão em concordância com o planejamento estratégico e incremento de qualidade nas organizações hospitalares, foi criado em 2009 o Núcleo de Enfermeiro de Educação Permanente do Paraná (NEEP/ PR), com a finalidade de construir conhecimentos nas áreas de educação continuada e permanente em saúde, a partir de discussões científicas, criação e consolidação de um grupo de estudos.

Diante do exposto, este estudo teve por objetivo descrever a trajetória do NEEP/PR e sua contribuição na institucionalização da educação permanente nas organizações hospitalares, desde sua fundação até os dias atuais.

A relevância da realização deste estudo pauta-se na contribuição da compreensão do caminhar da educação continuada à educação permanente nas organizações hospitalares do município de Curitiba com membros representados no NEEP/PR.

\section{- MÉTODO}

Trata-se de uma pesquisa histórico-social, que examina a dimensão de uma sociedade, neste caso dirigida sua atenção para um grupo profissional(6), realizada entre o período de março de 2013 e janeiro de 2014.

Para coleta de dados foram utilizados como fontes históricas os seguintes documentos: 56 atas de reuniões, dois regimentos internos escritos, um estatuto, quatro relatórios de avaliação de eventos denominados fóruns, que se encontravam arquivados no acervo do NEEP/PR, datados desde sua fundação em setembro de 2009 até janeiro de 2014.

Destas fontes foram extraídos os dados para a análise e interpretação, os quais foram organizados e interpretados segundo o objetivo da investigação proposta. Essa etapa consistiu num processo de síntese e de inferências sobre as informações contidas nos documentos analisados, desvelando seu conteúdo manifesto e latente ${ }^{(7)}$.

No que diz respeito aos aspectos éticos-legais, este estudo obteve aprovação em novembro de 2012 do Comitê de Ética em Pesquisa de uma universidade do sul do Brasil sob o CAAE: 06903612.8.0000.0102. 
A estrutura organizacional do NEEP/PR é composta de: um Presidente, um Vice-presidente, um Tesoureiro, um Secretário e Membros Efetivos, tratam-se de enfermeiros que atuam em serviços de educação permanente de instituições de saúde, com membresia comprovada mediante participação em três reuniões consecutivas comprovadas em ata ${ }^{(8)}$.

Esta estrutura foi mais bem definida após a realização da revisão e reestruturação do seu Regimento Interno, cuja aprovação foi deferida em reunião extraordinária (número 001/2013) pelos membros efetivos no dia 31 de janeiro de 2013. O Regimento consolidou o NEEP/PR como um grupo, sem fins lucrativos e independente, de enfermeiros responsáveis pelos serviços de educação continuada/ permanente de organizações hospitalares do Paraná( ${ }^{(8)}$. Desde a sua fundação, contou com participação de 42 enfermeiros de serviço de educação permanente de organizações hospitalares privadas, públicas e filantrópicas. Até janeiro de 2014 contava com 18 membros efetivos.

O percurso histórico-social do NEEP/PR parte de uma nova lógica da gestão de pessoas para a educação e o desenvolvimento profissional, impulsionada pelo Sistema Único de Saúde, pela instituição da Política Nacional de Educação Permanente, elevação da escolaridade, pelas Diretrizes Curriculares Nacionais, inovações tecnológicas, e pelos processos de certificação de qualidade nas organizações de saúde, que desvelaram a necessidade de ressignificar os processos educativos no contexto laboral das organizações hospitalares ${ }^{(9-10)}$.

Inicialmente denominado "Núcleo de Enfermeiros de Educação Continuada de Curitiba (NEEC/ Curitiba)", depois nominado de Núcleo de Enfermeiros de Educação Continuada do Paraná - NEEP/ PR, foi criado em 14 de setembro de 2009. No início do ano de 2009, três enfermeiras de organizações hospitalares distintas, reuniram-se e com o objetivo de fomentar o intercâmbio da educação no contexto dessas organizações na cidade de Curitiba. Para tanto, propuseram estruturar e elaborar um evento científico.

Esse evento ocorreu em julho do referido ano e foi nominado primeiro Fórum de Educação Continuada em Enfermagem de Curitiba sob o tema "Apresentação dos serviços de educação continuada de Curitiba", com o apoio de um hospital filantrópico da cidade, onde houve mobilização para formação de um núcleo de enfermeiros de educação continuada.

Num primeiro momento, os Fóruns se tratavam de estratégias efetivas para aproximar os profissionais enfermeiros e direcionar modelos eficazes de educação continuada, bem como se tornaram espaços para promover discussões, atualizações, aprendizagem de métodos de ensino e demais temas relativos ao assunto.

Em 05 de novembro de 2009, na realização do segundo Fórum de Educação Continuada em Enfermagem de Curitiba, sob o tema "Levantamento da Necessidade de Treinamentos", o evento contou com a participação de quarenta e um ouvintes. Neste fórum ocorreu abertura para filiação de novos membros com o intuito de criar um grupo independente de enfermeiros que atuavam em serviços de educação continuada de organizações hospitalares, a fim de promover discussões relacionadas à Educação Continuada em Enfermagem e aos desafios emergentes para os enfermeiros que atuavam nessa área.

Nesse segundo Fórum, também, ficou evidente a existência de um grande campo a ser conquistado pelos serviços de educação continuada e de sua importância para capacitação e atualização de profissionais da saúde. Frente ao exposto, emergiu a necessidade de os serviços de educação continuada discutirem amplamente os avanços na área de educação nas organizações hospitalares, para trabalhar de maneira mais efetiva, direcionados pela missão, visão e valores de cada instituição, com vistas à melhoria da qualidade da assistência.

Ressalta-se que as definições de Educação Continuada utilizadas e discutidas na avaliação do segundo Fórum, começaram a se aproximar do conceito da Educação Permanente, quando os membros do núcleo colocaram que a Educação Continuada não se restringia apenas à aprendizagem de sala de aula, em locais formais, mas também se desenvolvia de forma crítico-reflexiva, considerando o local de trabalho e situações do próprio cotidiano, a fim de alcançar um aprendizado diferenciado daquele 
da educação tradicional(2,9).

Destaca-se aqui que em dezembro de 2009 foi estruturado e aprovado o primeiro Estatuto do então "Núcleo de Enfermeiros de Educação Continuada de Curitiba (NEEC/Curitiba)", o qual definiu a estrutura hierárquica do núcleo, definindo as atribuições do Presidente, Vice-presidente, Secretário e demais membros, os quais só podem ser enfermeiros que atuam em serviços de educação continuada das organizações. Trata-se de uma estrutura hierárquica transversal que se mantém até os dias atuais.

No ano de 2010 ocorreu aumento do número de membros, passando de uma média de seis participantes por reunião, para uma média de dez participantes. Foi realizado um total de sete reuniões ordinárias em 2010. Nestas reuniões as discussões envolveram a realização do terceiro e quarto Fóruns de Educação Continuada sob os temas "O papel da educação continuada no processo de acreditação hospitalar" e "O papel da educação continuada nas instituições de saúde", respectivamente; bem como a responsabilidade legal do enfermeiro de educação continuada atuar em práticas educativa sem uma perspectiva multiprofissional.

Em relação ao processo de acreditação hospitalar, as discussões foram centradas na recomendação da Organização Mundial de Saúde (OMS), que propõe aprendizagem consubstanciada no valor que se dá a uma realidade em face às necessidades de saúde das pessoas e das populações, a partir da análise dos determinantes sociais e econômicos, mas, sobretudo, de valores e conceitos dos profissionais. Nesse sentido, a educação permanente é importante por permitir a construção de relações e processos que vão das equipes em atuação às práticas institucionais ${ }^{(11)}$.

Quanto à responsabilidade legal do enfermeiro de educação, as discussões foram alicerçadas no referencial da OPAS que recomenda um profissional da área da saúde para ser o coordenador e responsável pelo setor de educação e desenvolvimento de pessoas nos serviços de saúde, com dedicação exclusiva e diretamente envolvido com o atendimento das necessidades de desenvolvimento pessoal e profissional. Nestas organizações, é o profissional enfermeiro que assume tal coordenação, visto que possui competência para assumir posição estratégica para a estruturação de um sistema de gerenciamento e ensino compatíveis com as exigências e demandas institucionais ${ }^{(3)}$.

Outro tema amplamente discutido nas reuniões ordinárias no ano de 2010 foram os processos de ensino e aprendizagem utilizados pelos serviços de educação das organizações hospitalares, sustentado pelo referencial da Política Nacional da Educação Permanente. Ficou evidente a necessidade repensar estes processos. Os membros do NEEP/PR externaram sua insatisfação sobre algumas ações realizadas para o treinamento e desenvolvimento dos recursos humanos em determinadas organizações hospitalares, sustentadas pela lógica do modelo escolar ou acadêmico, centralizado na atualização, geralmente com enfoque disciplinar, em ambiente didático e baseado em técnicas de transmissão de conhecimentos $^{(9)}$.

Diante do exposto, pode-se afirmar que os serviços de educação começaram a perceber que a Educação Continuada, quando tem seu investimento na capacitação dos recursos humanos, sobretudo na aplicação técnica, não reflete obrigatoriamente em mudança do comportamento humano, em especial quando sua metodologia se pauta na pedagogia da transmissão, na lógica do modelo escolar e seus resultados na apropriação. Trata-se de uma abordagem de ensino-aprendizagem que não interfere de forma satisfatória na qualidade da produção dos serviços prestados em saúde, pois não repercute na transformação da realidade.

Estes questionamentos acarretaram séria renovação nos enfoques e estratégias da capacitação do pessoal da saúde, atualmente relacionados à Educação Permanente, fruto da visão de que o conhecimento não se "transmite", mas se constrói a partir das dúvidas e do questionamento das práticas vigentes à luz dos problemas contextuais ${ }^{(9-10)}$.

Ao final do ano de 2010, o estatuto do núcleo foi reestruturado e em reunião ordinária ocorrida no dia 02 de dezembro, foi aprovado o Regimento Interno do NEEC/Curitiba, em substituição a esse estatuto, que definiu o núcleo como um Grupo Especialista de Assessoria ao Serviço de Educação Continuada dos Serviços de Saúde, com a finalidade de unificar as práticas em educação continuada, bem como promover momentos de discussão científica e fortalecimento da categoria profissional.

Nesse mesmo período ficou estabelecido um cronograma mensal de reuniões e a periodicidade dos 
Fóruns passou de trimestral para anual. Os membros do núcleo perceberam que as reuniões mensais tornaram-se espaço livre para discussões e reflexões, bem como um lugar sem conflitos de interesse, que favorecia a produção de conhecimento e a troca de experiências. No que se referem aos Fóruns, estes passaram a ser um espaço de debate, aberto à comunidade profissional e acadêmica, com vistas à socialização de conhecimentos amplamente discutidos e construídos nas reuniões ordinárias mensais.

No ano de 2011 foi realizado um total de seis reuniões ordinárias. Das temáticas contempladas nas pautas dessas reuniões e discutidas pelos membros do núcleo, destacaram-se: o uso de indicadores de qualidade específicos para avaliação da eficácia dos treinamentos e a atuação do enfermeiro enquanto líder e, por conseguinte, educador de sua equipe.

Em relação aos indicadores de qualidade, todos os membros apresentaram e discutiram sobre quais utilizavam nas organizações hospitalares em que trabalhavam, sendo que a grande maioria tinha como indicadores: horas de treinamento por colaborador e por setor e avaliação de eficácia de treinamento. Essa demanda surgiu a partir dos processos de certificação de qualidade que estas organizações sofreram, em sua grande maioria, nos anos de 2010 e 2011.

Pode-se inferir que houve consenso sobre a utilização de indicadores de qualidade. Já é prática nas organizações, porém ainda é necessário implementar estratégias de análise desses indicadores para que sejam passíveis de comparabilidade e reflitam os diferentes contextos da assistência a saúde prestada, a fim de sustentar programas e políticas públicas de saúde e educação com vistas à melhoria da qualidade assistencial ${ }^{(12)}$.

A outra temática, além de ser ricamente discutida na reunião, sobre a atuação do enfermeiro líder e educador culminou no tema central do quinto fórum de Educação Continuada: "O impacto do acompanhamento do líder no desenvolvimento da equipe", visto que a liderança é, dentre as competências gerenciais, a mais requerida ao processo de trabalho do enfermeiro nas organizações hospitalares. Com base nos debates produzidos nesse Fórum, concluiu-se que somente pode ser líder de uma equipe o profissional que promove seu auto desenvolvimento, estabelece metas e estratégias para seu crescimento profissional, assim como de seus liderados, com vistas a fortalecer as potencialidades da equipe e superar suas fragilidades ${ }^{(10,12)}$.

Em 2012, foram realizadas um total de onze reuniões ordinárias e houve a ampliação do número de participantes para uma média de 12. Essas reuniões tiveram como principais temas de discussão e estudo: a falta de adesão do enfermeiro nos processos de educação na integração do novo colaborador e a necessidade de formação específica ao enfermeiro que atua na área de educação nas organizações hospitalares.

No que se refere à formação específica do enfermeiro de educação, tornou-se visível à necessidade de desenvolvimento de competências que necessitam ser mobilizadas pelo profissional que exerce a função de coordenador de um serviço de educação. Com base nos debates realizados nas reuniões do núcleo, evidenciou-se a importância e a premência de definir novos mecanismos, instrumentos, modalidades e estratégias para oaperfeiçoamento das competências destes enfermeiros coordenadores, a fim de contribuir para uma postura reflexiva, com a determinação da gestão com competências na prática profissional de enfermagem e com o crescimento destes profissionais no mundo do trabalho atual ${ }^{(13)}$.

A falta de adesão do enfermeiro nos processos de educação na integração do novo colaborador foi a temática do sexto Fórum de Educação Continuada em Enfermagem sob o título "O papel do enfermeiro diante da integração do novo colaborador". Através de exposições realizadas no instrumento de avaliação respondido pelos participantes do evento, foi considerado imperativo que o enfermeiro discuta e repense o seu papel de educador, bem como compreenda a educação permanente como um recurso que facilitará a integração e permanência do novo colaborador, pois impulsiona a autonomia, o crescimento pessoal, profissional e, consequentemente, o organizacional ${ }^{(14)}$.

Ao final do ano de 2012 ocorreu a mudança da presidência prevista no Regimento Interno do NEEC Curitiba que, em conjunto com os membros efetivos, propôs revisão e atualização desse regimento; e, mudança do nome para NEEP/PR, sustentada pelas mudanças ocorridas na área de educação nas organizações hospitalares nos últimos anos e pela Política Nacional de Educação Permanente. Os membros do núcleo passaram a compreender a educação como aquela que se desenvolve no trabalho, 
pelo trabalho e para o trabalho, sendo uma das modalidades de educação no contexto laboral ${ }^{(9)}$.

O novo Regimento Interno foi mais bem descrito e aprovado na primeira reunião mensal do ano de 2013. Com a atualização e revisão do seu Regimento, o NEEP/PR firma sua condição de um grupo que produz conhecimento e passa a acolher as particularidades institucionais e as necessidades de formação e desenvolvimento dos profissionais da área de saúde e a capacidade já instalada nas organizações, para nortear as discussões científicas e do grupo de estudo capazes de refletir em ações de educação para os recursos humanos na área de saúde das organizações hospitalares ${ }^{(8)}$.

Essas ações de educação fundamentam-se na lógica da educação permanente que, mais do que atualizar, vem para ensinar a problematizar, questionar, repensar e reinventar o cotidiano dos serviços de saúde. Pois utiliza a Metodologia da Problematização e a Aprendizagem Significativa nos processos educativos de trabalhadores de saúde para a melhoria da qualidade dos serviços e a transformação das práticas de saúde ${ }^{(11)}$. O que é possível é sensibilizar os indivíduos e estimulá-los a pensar de maneira reflexiva, a fim de que adquiram uma visão crítica da realidade que os cerca ${ }^{(15)}$.

Nesta perspectiva, o ano de 2013 caracterizou-se por um ano de mudanças significativas. Além da revisão e atualização do Regimento Interno, que envolveu três alterações: (1) na Estrutura Organizacional, considerando os cargos de vice-presidente e tesoureiro; (2) Definição do Conselho Técnico-Administrativo; e, (3) Descrição detalhada das atribuições desse conselho, ocorreu, também, a alteração da identidade visual do NEEP/PR; a criação de uma página na Internet; e, participação em pesquisa científica, sendo cenário de estudo de uma dissertação de mestrado de um de seus membros. No ano de 2014, estas mudanças fortaleceram o grupo criando um sentimento de pertencimento ao núcleo e, ao mesmo tempo de reconhecimento do NEEP/PR, como um espaço efetivo para discussão e reflexão-crítica na construção de saberes capazes de provocar mudanças nas organizações hospitalares.

\section{- CONTRIBUIÇÕES DO NEEP/PR NA INSTITUCIONALIZAÇÃO DA EDUCAÇÃO PERMANENTE NAS ORGANIZAÇÕES HOSPITALARES}

De acordo com os referencias teóricos trabalhados, as reflexões e os debates que permearam as discussões tanto das reuniões quanto dos Fóruns realizados pelo NEEP/PR ao longo destes quatro anos, pode-se afirmar que os membros deste núcleo consideram a educação como uma ferramenta para mudanças e transformações. As transformações sociais e educacionais têm repercussões nas formas de produzir, nos distintos campos do saber e de produção de bens e serviços ${ }^{(2)}$.

Percebeu-se, então, que a educação passa a consumir cada vez mais espaço na vida cotidiana das pessoas, em um mundo onde a rapidez das transformações se conjectura com o fenômeno da globalização e elevação do grau de participação dos indivíduos nas sociedades modernas. Por conseguinte, a evolução rápida do mundo requer contínuas e constantes atualizações dos conhecimentos $^{(16-17)}$.

Dessa forma, o NEEP/PR compreendeu que qualquer organização hospitalar que pretenda ser eficiente e progressista necessita melhorar de forma qualitativa. Para isso necessita de profissionais orientados e competentes e, pelo processo de educação permanente, ampliar e aperfeiçoar os saberes destes indivíduos. Assim, a Educação Permanente em Saúde torna-se um importante instrumento capaz de contribuir na mudança do cenário e garantir aos trabalhadores e usuários o seu papel de protagonistas do sistema de saúde ${ }^{(18)}$.

No ano de 2013, houve a necessidade de melhor compreender e ampliar as discussões sobre as questões que envolvem a educação permanente na área da saúde, a fim de entender que esse não é um processo atual. A educação no contexto das organizações hospitalares vem sofrendo atualizações ao longo dos tempos, na busca de melhoria da qualidade dos serviços de saúde.

Contemporaneamente nas organizações hospitalares, ainda existe a necessidade de capacitar seus profissionais por meio de uma série de atividades genericamente denominadas de treinamentos e cursos emergenciais ou pontuais, estruturados e contínuos. Estes treinamentos corroboram com a lógica tecnicista do saber-fazer em detrimento do saber-ser, ou seja, às práticas simplistas de admitir e demitir os indivíduos ${ }^{(9)}$. 
Assim, compreende-se que a educação continuada persiste nas organizações hospitalares, orientada para o preparo de determinada função e melhor desempenho do profissional com ênfase em treinamentos, reciclagens, palestras e cursos, tendo em vista a evolução tecnológica e científica, a satisfação da clientela e a imagem da instituição ${ }^{(19)}$.

Contudo, a partir das reflexões e produção saberes desenvolvidas junto ao NEEP/PR, há uma tendência para o investimento na capacitação dos profissionais com vistas à mudança do comportamento humano e, consequentemente, na melhoria da assistência em saúde prestada. Com isto, ao lado da educação no trabalho, concentrada em treinamentos e atualizações técnicas, algumas transformações de maior impacto estão sendo introduzidas na capacitação de pessoal em saúde e enfermagem nas organizações hospitalares ${ }^{(20)}$. Em especial, propostas que têm por objetivo aproximar educação e trabalho gerados como práticas sociais. Estas propostas alinham-se ao conceito de Educação Permanente ${ }^{(9,17)}$.

A partir destas colocações, pode-se afirmar que os membros do NEEP/PR começaram a perceber que a Educação Continuada, cujo investimento na capacitação dos recursos humanos, sobretudo na aplicação técnica, não reflete obrigatoriamente na mudança do comportamento humano, uma vez que a sua metodologia se pauta na pedagogia da transmissão e seus resultados na apropriação. Assim, essa abordagem de ensino-aprendizagem não interfere de forma satisfatória na qualidade da produção dos serviços prestados em saúde, pois não repercute na transformação da realidade ${ }^{(19)}$.

Diante do exposto, ocorreu a ampliação dos horizontes para a lógica da educação permanente pelo NEEP/PR e, por conseguinte, nas organizações hospitalares, na direção da integralidade, para as ações da assistência/cuidado, a fim de contribuir para a articulação de estratégias da equipe multiprofissional na resolutividade de problemas do paciente, facilitando a transformação das práticas de ensinoaprendizagem na produção do conhecimento.

Com isso, a educação permanente nas organizações hospitalares começa a ser entendida como ferramenta essencial ao processo de qualificação, pois utiliza como princípios: competência profissional, motivação organizacional e avaliação contínua, os quais serão alcançados a partir de uma transformação do profissional de saúde para atuar de forma crítica e reflexiva no seu cotidiano ${ }^{(11,16)}$.

Nessa perspectiva, o NEEP/PR compreendeu que os serviços de educação permanente nas organizações hospitalares possuem a incumbência de, além de providenciar suporte organizacional e administrativo, implantar uma cultura do trabalho de qualidade nas instituições hospitalares, a qual se inicia a partir de um planejamento estratégico de gestão com a participação ativa e dialógica dos profissionais dessas organizações ${ }^{(20)}$.

Destaca-se que, a partir da concepção que o NEEP/PR é um grupo de estudos que produz conhecimento, despertou também a compreensão da sua posição estratégica, bem como a do enfermeiro de educação permanente, que culminou na mudança da denominação de Serviços de Educação Continuada para Serviços ou Núcleos de Educação Permanente na maioria das organizações hospitalares representadas pelos participantes da pesquisa.

Assim, a abordagem da educação permanente nas organizações hospitalares que possuem membros no NEEP/PR passou de um plano operacional para um plano estratégico, com vistas à mudança das práticas de saúde e de enfermagem, no contexto da integralidade, do trabalho em equipe e da ampliação da cidadania e da autonomia dos indivíduos envolvidos nestas organizações.

\section{CONSIDERAÇÕES FINAIS}

O NEEP/PR constitui um espaço privilegiado pelo seu potencial em agregar formação científica, valorização profissional e construção de saberes. Trata-se de um importante grupo independente e sem fins lucrativos que busca construir e difundir os saberes relacionados à área de Educação Permanente em Saúde no contexto das organizações hospitalares.

Pode-se inferir que o presente estudo revelou que não somente é possível, mas é essencial investir nestes grupos de estudo, também, de discussão e formação, os quais têm como incentivos as práticas e as experiências de cada membro deste núcleo, que buscam primeiro a compreensão da realidade, 
para depois tentar transformá-la.

A valorização da trajetória histórica, da atuação e das contribuições do NEEP/PR é fundamental para os serviços de educação destas organizações. Ressalta-se que espaços como este possibilitam uma visão crítica acerca dos movimentos históricos e contemporâneos que envolvem a educação permanente nas áreas da saúde e da enfermagem, auxiliando na compreensão do presente de maneira reflexiva. Fornece ainda perspectivas futuras na construção de opções possíveis e desejáveis para o avanço de pesquisas relacionadas à temática deste estudo.

\section{REFERÊNCIAS}

1. Manzo BF, Brito MJM, Corrêa AR. Implications of hospital accreditation on the everyday lives of healthcare professionals. Rev. esc. enferm. USP. 46(2): 388-94.

2. Ceccim RB. Desenvolvimento de competências no trabalho em saúde: educação, áreas do conhecimento e profissões no caso da saúde. Tempus Actas de Saúde Colet. 2012; 6(2): 253-77.

3. Organização Panamericana de La Salud. Educación Contínua. Guia para la organización de programas de educación continua para personal de salud. Washington: División de Recursos Humanos e Investigación; 197.

4. Kurcgant P. Nurses' professional training [editorial]. Rev. esc. enferm. USP. 2011; 45(2): 307-8.

5. Kurgant P, organizadora. Gerenciamento em Enfermagem. 2.ed. Rio de Janeiro: Guanabara Koogan; 2012.

6. Barros JD. O campo da história. Especialidades e abordagens. 9.ed. Rio de Janeiro: Vozes; 2013.

7. Padilha MICS, Borenstein MS. O método da pesquisa histórica na enfermagem. Texto Contexto enferm. 2005; 14(4): 575-84.

8. Núcleo de Enfermeiros de Educação Permanente do Paraná. Regimento interno. 2012.

9. Davini MC. Enfoques, problemas e perspectivas na educação permanente dos recursos humanos de saúde. In: Brasil. Ministério da Saúde. Secretaria de Gestão do Trabalho e da Educação na Saúde. Departamento de Gestão da Educação em Saúde. Política Nacional de Educação Permanente em Saúde. Brasília; 2009.

10. Sade PMC, Peres AM. Development of managerial competencies of nurses by permanent education services. ATINER'S Conference Paper Series [Internet]. 2015; 1429 [acesso em 15 jun 2015]. Disponível: http://www.atiner. gr/papers/NUR2015-1429.pdf

11. Manzo BF, Ribeiro HCTC, Brito MJM, Alves M. Nursing in the hospital accreditation process: practice and implications in the work quotidian. Rev. Latino-Am. Enfermagem. 2012; 20(1): 151-8.

12. Rossaneis MA, Gabriel CS, Haddad MDCL, Costa-Melo MR, Bernardes A. Indicadores de qualidade utilizados nos serviços de enfermagem de hospitais de ensino. Rev. Eletr. Enf. [Internet]. 2014; 16(4) [acesso em 2015 Jul 02]. Disponível: http://www.revistas.ufg.br/index.php/fen/article/view/22956

13. Ekström L, Idvall E. Being a team leader: newly registered nurses relate their experiences. Journal ofnursing management [Internet].2015; 23(1) [acesso em 19 jun. 2015].Disponível: http://onlinelibrary.wiley.com/doi/10.1111/ jonm.12085/full

14. Salum NC, Prado ML. Continuing education in the development of competences in nurses. Texto Contexto Enferm. 2014; 23(2): 301-8.

15. Ribeiro JP, Rocha LP. Permanent education in health. An instrument to enhance interpersonal relations in nursing work. Invest. educ. enferm. 2012; 30(3): 412-7.

16. Freire P. Educação e mudança. Rio de Janeiro: Paz e Terra; 2001.

17. Miccas FL, Batista SHSS. Permanent education in health: a review. Rev Saúde Pública. 2014; 48(1): 170-85. 
18. Duarte MLC, de Oliveira AI. Compreensão dos coordenadores de serviços de saúde sobre educação permanente. Cogitare enferm. 2012; 17(3): 506-12.

19. Soto-Fuentes P, Reynaldos-Grandón K, Martínez-Santana D, Jerez-Yáñez O. Competencias para la enfermera/o en el ámbito de gestión y administración: desafíos actuales de la profesión. Aquichán. 2014; 14(1): 79-99.

20. Montanha D, Peduzzi M. Permanent education in nursing: survey to identify the necessities and the expected results based on the workers conception. Rev. esc. enferm. USP. 2010; 44(3): 597-604. 